\title{
El uso de metodologías activas en el aula: Aplicación de la metodología Scrum
}

\author{
Jordi Capó Vicedo ${ }^{a}$, Antonio Giménez-Morera ${ }^{b}$ \\ ${ }^{a}$ Universitat Politècnica de València, Centre for Research in Business Management (CEGEA), Spain. \\ jorcavi0@esp.upv.es, ${ }^{b}$ Universitat Politècnica de València, Department of Economy and Social \\ Sciences, Camino de Veras/n, 46022,Valencia, Spainangimo1@doctor.upv.es
}

\begin{abstract}
Resumen
En el presente artículo se detallan los resultados obtenidos de la aplicación de la metodología Scrum, basada en entregas parciales y regulares que ofrecen una alta agilidad, en asignaturas del Master Universitario en Dirección de Empresas que se imparte en la Escuela Politécnica de Alcoi de la Universitat Politècnica de València.
\end{abstract}

Palabras clave: Metodologías activas, enseñanza universitaria.

\section{Introducción}

Ante los profundos cambios que se están produciendo en la sociedad actual, la Universidad debe ser capaz de dar respuesta a las nuevas inquietudes que van surgiendo con el desarrollo y la aplicación de nuevas metodologías que permitan un aprendizaje permanente y la incorporación de competencias transversales a la formación del alumnado.

Una de las principales finalidades de la Universidad, es formar a sus alumnos para que adquieran una serie de competencias necesarias para su futuro profesional.

Por tanto, resulta evidente que uno de los ejes de la actividad universitaria es el aprendizaje de los estudiantes, y la tarea del profesor debe facilitarlo, proporcionando los factores favorables necesarios para que el alumno adquiera las competencias que le transmite el profesor.

En este punto, cabe destacar el cambio que está experimentando la universidad, puesto que se está pasando de un modelo más centrado en los profesores y en la enseñanza a un modelo centrado más en el alumno y en su aprendizaje, en el que el rol del profesor será el de organizar eficientemente su labor de aprendizaje. 
De este modo, será fundamental una adecuada elección de la metodología didáctica y pedagógica por parte del profesor, así como el planteamiento que realice de sus asignaturas con el fin de facilitar el aprendizaje de sus alumnos y los métodos y criterios de evaluación empleados.

En este contexto, resulta necesario plantearse cómo motivar a los alumnos para incrementar la participación, tomando como base la idea de que la motivación constituye un elemento clave para el aprendizaje.

En el presente artículo se detallan los resultados obtenidos de la aplicación de la metodología Scrum, basada en entregas parciales y regulares que ofrecen una alta agilidad, en asignaturas del Master Universitario en Dirección de Empresas que se imparte en la Escuela Politécnica de Alcoi de la Universitat Politècnica de València.

\section{Antecedentes}

Actualmente, y ante los profundos cambios que se están produciendo en los últimos años, las universidades se enfrentan a una nueva etapa en la que de nuevo deben de adaptarse a las exigencias y cambios que se están produciendo.

En este sentido el Libro Blanco sobre la Educación y la Formación de la Comisión Europea, precisa cuales han sido los tres grandes impactos de nuestro tiempo sobre el mundo de la educación: la emergencia de la sociedad de la información que está transformando la naturaleza del trabajo y de la organización de la producción; el fenómeno de la mundialización que incide sobre las posibilidades de creación de empleo, y, finalmente, la revolución científico-técnica, que crea una nueva cultura y que plantea acuciantes cuestiones éticas y sociales.

Todos estos cambios que se están produciendo (globalización, cambios demográficos,...) junto con el paso de una sociedad industrial a la llamada sociedad del conocimiento han provocado la necesidad de adaptarse y de adoptar una serie de cambios en el ámbito universitario. Tal y como subraya Perron (2008), si antes lo importante era transmitir a los estudiantes los fundamentos de una asignatura como, por ejemplo, la física, la química, la psicología, la literatura o el derecho, de tal forma que pudieran construir con ello toda su vida profesional y continuar formándose de forma autodidacta, hoy se exige más bien "flexibilidad" y un "aprendizaje durante toda la vida".

En este sentido desde el punto de vista docente, con la aparición de las nuevas tecnologías de la información y de la comunicación (TIC) el nivel de conocimiento y la forma de adquirirlo cambia cada vez más rápidamente. Es necesario, por tanto, practicar y adquirir 
capacidades que puede que no estén relacionadas directamente con la materia o que sean multidisciplinares, puesto que el papel del docente cambia y, en vez de ser quien centra la información, pasa a ser un guía, un tutor y un orientador para el alumno y su aprendizaje (Casas, 2005).

\subsection{La asignatura Responsabilidad Social Corporativa}

La asignatura de Responsabilidad Social Corporativa se enmarca dentro del segundo curso del máster oficial en dirección de empresas que se imparte en el campus de Alcoi de la Universitat Politècnica de València. Es una asignatura optativa de 4,5 créditos del itinerario de dirección corporativa correspondiente al primer cuatrimestre.

Hay que tener en cuenta que se trata de una asignatura de máster y que por tanto los alumnos ya han realizado estudios superiores y por tanto tienen una experiencia universitaria considerable.

Los objetivos de la asignatura son por una parte conocer los conceptos básicos y las herramientas de Responsabilidad Social Corporativa (RSC) presentes en el mundo empresarial y por otra aportar los conocimientos teóricos y prácticos para la gestión de una empresa que quiera ser socialmente responsable.

Hay que tener en cuenta que las empresas cada vez son más conscientes de que, además de la presión que se ejerce desde los distintos grupos de interés y desde las administraciones públicas para que asuman sus responsabilidades, así como una mayor transparencia en sus prácticas empresariales, la integración de la Responsabilidad Social Corporativa en sus estrategias empresariales puede suponer una serie de ventajas y de beneficios a largo plazo. Es en este punto dónde se hace más hincapié en la asignatura, con el fin de que los alumnos sean capaces de ver la importancia de este enfoque integrado de la gestión, lo que supone un modelo de gobierno basado en la sostenibilidad y la gestión de las externalidades empresariales en lo económico, social y medioambiental contempladas a un mismo nivel.

La asignatura se estructura en 6 unidades didácticas, con un enfoque eminentemente práctico, con clases participativas y la rrealización y análisis de casos prácticos complementarios a la teoría, así como de trabajos de búsqueda y análisis de información específica. 
Tabla 1. Datos descriptivos de la asignatura

\begin{tabular}{|c|c|c|c|}
\hline Asignatura & Titulación & Departamento & Alumnos \\
\hline $\begin{array}{c}\text { Responsabilidad Social } \\
\text { Corporativa }\end{array}$ & $\begin{array}{c}\text { Máster universitario en } \\
\text { Dirección de Empresas }\end{array}$ & $\begin{array}{c}\text { Economía y } \\
\text { Ciencias Sociales }\end{array}$ & 8 \\
\hline Centro & Créditos & Curso & Duración \\
\hline $\begin{array}{c}\text { Escuela Politécnica } \\
\text { Superior de Alcoi }\end{array}$ & 4,5 & $2^{\circ}$ & Cuatrimestral \\
\hline \hline
\end{tabular}

Fuente: Elaboración propia

\section{Metodología}

La metodología Scrum es un marco de trabajo utilizado para desarrollar y mantener productos complejos, basada en la teoría de control de procesos empírica.

Se trata de una metodología de desarrollo ágil en la que se llevarán a cabo una serie de ciclos breves para el desarrollo de los proyectos denominados "Sprints". Cada Sprint tiene un tiempo limitado, y se espera que al final de cada uno de ellos se haya generado una mayor funcionalidad del proyecto.

De acuerdo con los creadores de esta metodología, Schwaber y Sutherland, el marco de trabajo Scrum consiste en los Equipos Scrum y sus roles, eventos, artefactos y reglas asociadas. Dentro de este marco de trabajo, existen una serie de reglas mediante las cuáles se relacionan todos los componentes de la misma.

Dentro del marco de trabajo, el equipo Scrum se compone de un responsable del producto (dueño del producto, Product Owner), el equipo de desarrollo (Development Team o Scrum Team) y el Scrum Master, que es el responsable de asegurar que todo se entiende y se aplica de forma correcta.

Por otra parte en cada Sprint tienen lugar una serie de eventos:

- En primer lugar se realiza una reunión de planificación de Sprint (esta reunión se debe realizar al inicio de cada Sprint) en la que se marca el objetivo del Sprint, se organizan las diferentes tareas del equipo de forma que este trabaje de forma conjunta y no cada uno de forma individual.

- A continuación se realizan unas reuniones de seguimiento, normalmente se trata de reuniones cortas de 15-20 minutos que pueden ser bien diarias o en función de la disponibilidad del equipo se pueden fijar otros períodos. En estas reuniones los miembros del equipo de desarrollo plantea y contestan a las siguientes preguntas: 
¿Qué hice ayer que ayudó al Equipo de Desarrollo a lograr el Objetivo del Sprint?, ¿Qué haré hoy para ayudar al Equipo de Desarrollo a lograr el Objetivo del Sprint?, ¿Veo algún impedimento que evite que el Equipo de Desarrollo o yo logremos el Objetivo del Sprint?

- Al final del Sprint se realiza una reunión denominada de revisión en la que se analiza el incremento que se ha producido en el proyecto.

- Por último se lleva a cabo una reunión retrospectiva de Sprint en la que se evalúa el trabajo realizado y que permite elaborar un plan de mejoras para el siguiente Sprint.

Además de estos eventos, el Sprint se apoya en una serie de artefactos o elementos, mediante los cuales se puedan desarrollar los proyectos, en primer lugar una lista de producto en la que se recogen las características y todo lo que se podría necesitar en el producto, el responsable de esta lista es el Dueño del Producto (Product Owner) que es quién define el grado de prioridad entre ellas, denominada "Pila del producto" (Product Backlog).

En segundo lugar la denominada lista de pendientes del Sprint, o Pila del Sprint, en la que se definen los elementos de la lista de producto seleccionados para ese Sprint así como de todo el trabajo necesario para cumplir con los objetivos marcados.

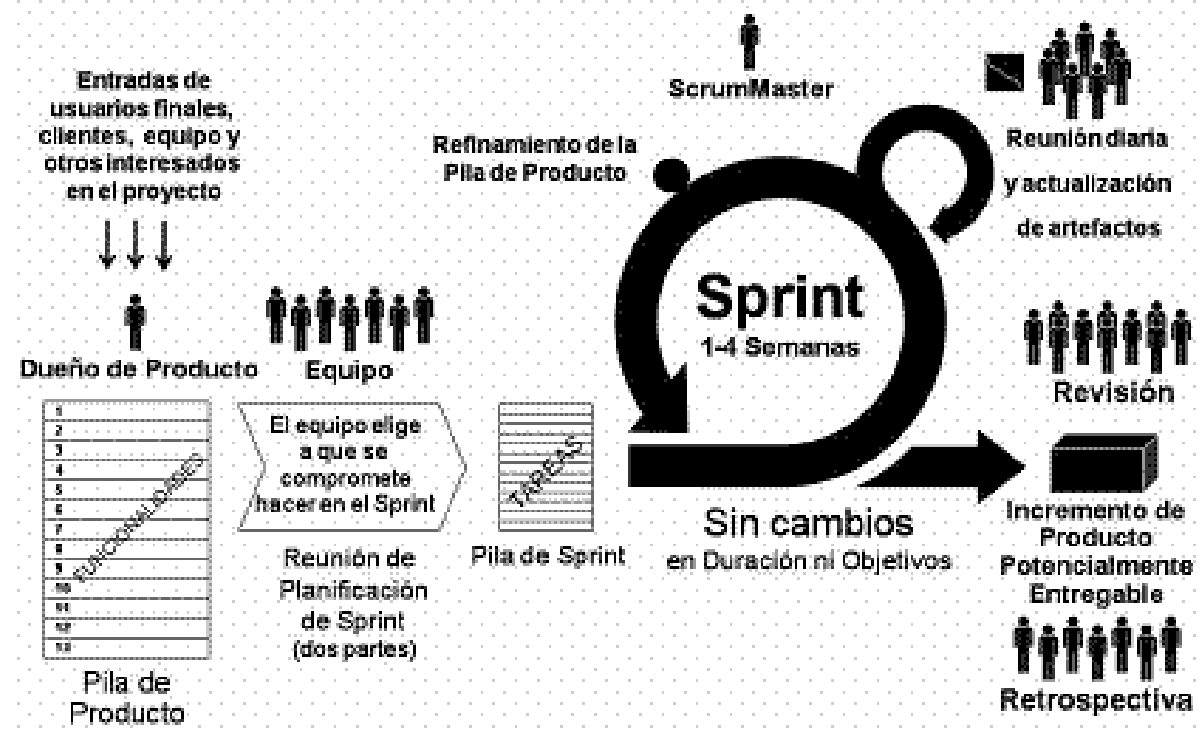

Figura 1. Roles, artefactos y eventos principales de SCRUM (Fuente: Deemer et al., 2009) 


\subsection{Aplicación de la metodología}

En muchas ocasiones a la hora de impartir una asignatura, se pueden dar situaciones en las que los alumnos con el transcurso de las clases pueden perder la motivación con respecto a la asignatura, lo cual puede conllevar también una pérdida de atención e incluso problemas de comportamiento por su parte.

Para intentar evitar estas situaciones, se planteó la aplicación de una nueva metodología que permitiera lograr un doble objetivo, por una parte motivar a los alumnos para que se involucraran en el funcionamiento de la asignatura, y por otra que vieran la relación de los contenidos vistos en clase con una aplicación práctica, y que por tanto incentivara su interés.

En primer lugar se realizó una reunión en la cuál se explicó en qué consistía la metodología Scrum, así como los objetivos que se pretendían alcanzar mediante su utilización en la asignatura.

Una vez que los alumnos ya supieron en qué consistía la metodología, se organizaron los equipos y se asignaron los diferentes roles en cada uno de los componentes de los equipos. En este punto se presentó la primera dificultad, puesto que al tratarse de una asignatura optativa de un máster, tan sólo habían 8 alumnos, por lo que se decidió hacer dos equipos de 4 personas.

Tras organizar los equipos y los roles se planteó el proyecto, consistente en analizar una empresa desde el punto de vista de la incorporación e integración de los principios de la RSC en su gestión y políticas y a partir de las conclusiones obtenidas, plantear una serie de mejoras y recomendaciones a implantar en las empresas dentro de las cuáles se enmarcaría la realización de una memoria de sostenibilidad.

Para la realización del proyecto se plantearon llevar a cabo 4 Sprints con una duración de tres semanas cada uno.

Las clases tenían una duración de 3 horas y se planteó la realización de las reuniones de revisión y retrospectivas de cada Sprint al inicio de las mismas y en presencia de todos los alumnos para de esta forma poder interactuar.

Además cada semana los alumnos se reunían y realizaban una reunión de seguimiento en la que ponían en común lo que habían realizado a lo largo de esa semana, se planteaban los objetivos que debían alcanzar en la próxima, así como si habían encontrado problemas para poder trabajar en la consecución de los objetivos que se habían marcado.

En la asignatura, cada vez que se finalizaba un Sprint, cada grupo preparaba un documento a partir de las reuniones de revisión de Sprint y de retrospectiva de Sprint que se habían 
realizado, con el fin de que se pudiera ir valorando la evolución del proyecto y la implicación de cada grupo.

Por último, en cuanto a la evaluación de la experiencia, dentro de la evaluación general de la asignatura, el proyecto supuso un $60 \%$ de la nota final en la que se valoró tanto la presentación de una memoria final y su exposición, como la participación de las diferentes fases (reuniones) del proyecto y la involucración de los miembros de cada equipo.

\section{Conclusiones}

En general la valoración de la experiencia por parte del alumnado ha sido muy buena. No obstante al principio hubo algunos problemas a la hora de identificar bien los roles y las misiones de cada alumno, por lo que se tuvo que volver a explicar y poner en común en la segunda reunión. Además en el caso en concreto de un grupo, uno de los miembros se quejó de las tareas asignadas puesto que entendía que no todos realizaban el mismo trabajo, en este caso se realizó una reunión con todos los integrantes del equipo y se planteó la cancelación del Sprint en el que estaban inmersos, sin embargo tras una puesta en común en la que todos interactuaron y expresaron sus opiniones, se procedió a una pequeña redistribución, tras la cuál todo mejoró considerablemente.

En las diversas reuniones que se mantuvieron durante el curso a excepción del caso anteriormente mencionado, todas las valoraciones y la actitud de los alumnos fue muy positiva.

Al final del curso se hizo una reunión final en la que los alumnos presentaron sus proyectos y sus impresiones acerca del trabajo realizado y de la metodología empleada, quedando de manifiesto que la mayoría de los alumnos habían tenido una mayor implicación y participación en la asignatura como consecuencia de la utiliación de esta metodología. 


\section{Referencias}

Casas, M. (2005). Nueva universidad ante la sociedad del conocimiento. Revista de Universidad y Sociedad del Conocimiento, 2 (2), 1-18.

Deemer, P., Benefield, G., Larmanas, C., Voode, B. (2009). Información básica de Scrum (The Scrum Primer). San $\quad$ Francisco. $\quad$ Recuperado de http://www.goodagile.com/Scrumprimer/Scrumprimer es.pdf

Oller, A., Gallardo, A., García, E., Valero, M. (2014). Uso de métodos ágiles y PBL en una asignatura de ingeniería del software del grado de ingeniería telemática. Gestión de proyectos de ingeniería del software en un entorno docente. Revista del Congrés Internacional de Docència Universitària i Innovació (CIDUI), 2, 1-12.

Perron, W. (2008). Cambio Cultural en la Universidad: ¿Motor o Peligro para el Desarrollo Universitario?. En Fundación Alexander von Humbolt (Ed.), XVII Encuentro Anual de la Asociación Alexander von Humboldt: Ciencia y sociedad: ejes de la transformación universitaria (pp. 35-64). Servicio Editorial de la Universidad del País Vasco.

Schwaber ,K.., Sutherland, J. (2013). The Scrum Guide. Recuperado de . https://www.Scrum.org/Scrum-Guide 